\title{
Effectiveness of bezafibrate and ursodeoxycholic acid in patients with primary biliary cholangitis: a meta-analysis of randomized controlled trials
}

\author{
Rohit Agrawala, Muhammad Majeed ${ }^{a}$, Bashar M. Attarc, Yazan Abu Omara, Chimezie Mbachia, \\ Yanting Wang ${ }^{a}$, Estefania Flores ${ }^{a}$, Shami Shaqib ${ }^{a}$, Yuchen Wang ${ }^{a}$, Victor Udechukwub, \\ Melchor Demetriac, Seema Gandhic
}

Cook County Health and Hospital System, Chicago, IL, USA

Abstract

Background Ursodeoxycholic acid (UDCA) and obeticholic acid are currently approved treatments
for primary biliary cholangitis (PBC). Since some patients do not respond adequately to UDCA,
other therapies, such as bezafibrate, have been developed. In this meta-analysis we evaluated the
efficacy and safety of using both UDCA and bezafibrate in patients with an inadequate response
to UDCA.

Methods We evaluated all randomized controlled trials comparing the combination of UDCA and bezafibrate with UDCA monotherapy. Standardized mean difference (SMD) was used to assess the treatment effect of combination therapy compared with UDCA alone.

Results Ten trials with a total of 369 patients were analyzed. UDCA and bezafibrate combination therapy was more effective than UDCA monotherapy in improving alanine aminotransferase (SMD -2.04, 95\% confidence interval [CI] -3.30 to -0.79), alkaline phosphatase at both less than 12 months (SMD -3.63, 95\%CI -6.43 to -0.84) and more than 12 months (SMD -2.33, 95\%CI -4.03 to -0.63 ), gamma-glutamyltransferase (SMD $-1.29,95 \% \mathrm{CI}-2.67$ to 0.08 ), triglyceride (SMD -0.80 , $95 \% \mathrm{CI}-1.41$ to -0.19 ), immunoglobulin M (SMD -1.48, 95\%CI -2.39 to -0.56 ), and cholesterol (SMD -4.61, 95\%CI -7.34 to -1.89). There was no difference between the 2 groups in bilirubin, aspartate aminotransferase or albumin. None of the adverse effects differed statistically between the 2 groups.

Conclusion UDCA and bezafibrate combined treatment is superior to UDCA alone in UDCA non-responders with regard to decreasing liver biochemistry markers, without any significant increase in side effects in patients with PBC.

Keywords Primary biliary cholangitis, ursodeoxycholic acid, bezafibrate, combination therapy

Ann Gastroenterol 2019; 32 (5): 1-9

Department of a Medicine (Rohit Agrawal, Muhammad Majeed, Yazan Abu Omar, Chimezie Mbachi, Yanting Wang, Estefania Flores, Shami Shaqib, Yuchen Wang, Melchor Demetria); ${ }^{b}$ Hospital Medicine (Victor Udechukwu); 'Division of Gastroenterology and Hepatology, Department of Medicine (Bashar M. Attar, Melchor Demetria, Seema Gandhi), Cook County Health and Hospital System, County, Chicago, IL, USA

Conflict of Interest: None

Correspondence to: Rohit Agrawal, MD, Department of Medicine, Cook County Health and Hospital System, IL, USA, e-mail: rohit.agrawal@cookcountyhhs.org

Received 9 April 2019; accepted 20 June 2019; published online

DOI: https://doi.org/10.20524/aog.2019.0403

\section{Introduction}

Primary biliary cholangitis (PBC) is an autoimmunemediated liver disease characterized by a triad of chronic cholestasis, anti-mitochondrial antibody (AMA) positivity, and liver biopsy findings of non-suppurative destructive cholangitis and interlobular bile duct destruction. It can eventually progress to cirrhosis [1,2]. Ursodeoxycholic acid (UDCA), the first and most widely used medication for PBC has shown to improve biochemical markers and improve transplant-free survival. However, $15 \%$ of such patients who biochemically respond to UDCA eventually develop hepatic complications [3-7]. A recent study estimated that around $20 \%$ of patients treated with UDCA show an inadequate response [6]. Compared to responders to 
UDCA, these patients are at a greater risk of hepatic complications such as ascites, variceal bleeding and hepatic encephalopathy, as well as a $25 \%$ greater risk of liver transplantation [6,7]. In 2016, the Food and Drug Administration approved obeticholic acid for use in combination with UDCA in PBC patients who showed an inadequate response to UDCA. Obeticholic acid is also approved as monotherapy in patients unable to tolerate UDCA because of side effects; however, it is associated with pruritus, which can be a significant nuisance for the patient. There is limited research on the long-term effects of obeticholic acid $[8,9]$.

Bezafibrate, a hypolipidemic fibrate currently approved for PBC treatment in France, has been shown to be beneficial when used in combination with UDCA in patients who have an inadequate response to UDCA alone [10]. It has known antiinflammatory, anti-fibrotic and anti-cholestatic effects, yet its mechanism remains unclear in PBC. It is believed that the benefits are driven by its activation of the dual peroxisome proliferator activator receptor (PPAR) and pregnane X receptor agonist [11].

Several trials have addressed the effects of combination therapy in such patients; however, a major limitation of all these trials was their sample size. The most recent trial by Corpechot et al, the largest study on bezafibrate, included 100 patients [12]. We therefore performed a meta-analysis to assess the efficacy of combination therapy with bezafibrate and UDCA compared to UDCA alone in PBC patients who had shown an inadequate response to UDCA monotherapy.

\section{Patients and methods}

\section{Search methodology}

We performed this meta-analysis in accordance with the Preferred Reporting Items of Systematic Review and MetaAnalysis (PRISMA) guidelines [13]. We searched MEDLINE, EMBASE, Ovid, Scopus and the Cochrane Library (Cochrane Central Register of Controlled Trials and Cochrane Database of Systematic Reviews) from inception to June 2019 to identify published articles analyzing the use of ursodeoxycholic acid and bezafibrate in PBC. No language or date limitations were imposed. The keywords used in combination were "randomized controlled trial", "bezafibrate", "ursodeoxycholic acid", "primary biliary cholangitis", "primary biliary cirrhosis", and "fenofibrate". In order to identify additional studies, the reference list of all eligible studies was reviewed. Proceedings of major scientific conferences were also searched to identify grey literature.

\section{Inclusion criteria}

We included only randomized controlled trials (RCT) that compared combination therapy with UDCA and bezafibrate to UDCA monotherapy. The study population comprised patients with $\mathrm{PBC}$, diagnosed according to the presence of any 2 of the following criteria: 1) biopsy of the liver consistent with PBC; 2) biochemical evidence of cholestasis in absence of obstruction; and 3) presence of AMA.
Articles were excluded if they did not refer to RCT or if no outcomes were reported. Two reviewers independently performed study selection according to the eligibility criteria. Disagreements were resolved by discussion with a third reviewer (SS).

\section{Data extraction and assessment of study quality}

The following data were independently abstracted into a standardized form: study characteristics (study design, primary author, year of publication, and country of the population studied), characteristics of the study population (total number of patients in monotherapy and combination therapy group, age of patients, dose of UDCA and bezafibrate used), change in biochemical markers (triglycerides [TG], immunoglobulin M [IgM], alanine aminotransferase [ALT], aspartate aminotransferase [AST], gamma-glutamyltransferase [GGT], alkaline phosphatase [ALP], albumin, total bilirubin [TB], total cholesterol, creatinine kinase $[\mathrm{CK}]$ ) and side effects of therapy (reactive pruritus, myalgia, arthralgia, CK elevation). The revised Cochrane risk of bias tool (RoB 2) for RCTs was used to assess the quality of published trials [14].

\section{Outcome definition}

The primary outcome of this meta-analysis was defined as a change in biochemical markers such as ALT, AST, GGT, IgM, $\mathrm{TB}$, and albumin. The secondary outcomes included mortality and the rate of adverse effects, including myalgias, reactive pruritus, and elevation of CK.

\section{Statistical analysis}

STATA (Version 14.0, College station, TX) was used for analysis. Continuous data were pooled to calculate standardized mean differences (SMD), while dichotomous outcomes were expressed as odds ratio (OR) with $95 \%$ confidence interval (CI) for both, using a random-effects model with inverse variance weighting. Heterogeneity across studies was evaluated using the Higgins $I^{2}$ statistic, where values of $25-50 \%, 50-75 \%$ and $>75 \%$ represent mild, moderate and severe heterogeneity, respectively. Egger's regression test was used to test for publication bias. A P-value $<0.05$ was considered significant in all cases.

\section{Results}

Literature search results, study characteristics, and quality assessment

Our search strategy retrieved a total of 211 published articles. Among these, 10 RCTs comprising 369 patients 
were included in our analysis. The baseline characteristics of the trials are listed in Table 1. The PRISMA flow chart (Fig. 1) summarizes the results of our literature review. Only 1 of the 10 trials was a double-blinded study. Nine of the 10 trials included in our meta-analysis were considered to have a high risk of bias (Fig. 2,3). Patient with inadequate biochemical response to UDCA therapy defined variably in different studies as per Barcelona criteria, Paris- 2 criteria or study specific criteria were included in the study. The patients mean age ranged from 53-64 years and the mean follow-up interval from 3-24 months. The daily dose of bezafibrate used was $400 \mathrm{mg} /$ day, while the UDCA daily dose ranged from 600$1500 \mathrm{mg}$ daily.

\section{Results of meta-analysis}

The summarized results of our meta-analysis are presented in Table 2 and Fig. 4,5.

\section{Bilirubin}

A change in serum bilirubin was mentioned in 5 trials that included 164 patients. There was no statistically significant difference between the two groups (SMD -0.77, 95\%CI -2.20 to $0.65 ; \mathrm{P}=0.287)$ and there was severe heterogeneity $(\mathrm{P}<0.001$, $\left.I^{2}=92.6 \%\right)($ Fig. $4 \mathrm{~A})$.

\section{ALT}

Three trials that included 142 patients reported a change in ALT level from baseline. Combination therapy was associated with an extra decline in ALT level compared to monotherapy in patients with $\mathrm{PBC}$ (SMD $-2.04,95 \% \mathrm{CI}-3.30$ to -0.79 ; $\mathrm{P}=0.001)$. There was severe heterogeneity $\left(\mathrm{P}=0.001, I^{2}=85.6 \%\right)$ (Fig. 4B).

\section{AST}

Three trials with 128 patients compared changes in AST between monotherapy and combination therapy groups. There was no statistical difference between these groups (SMD $-0.34,95 \% \mathrm{CI}-1.76$ to $1.08 ; \mathrm{P}=0.639$ ) and there was severe heterogeneity $\left(\mathrm{P}<0.001, I^{2}=90.5 \%\right)$ (Fig. $\left.4 \mathrm{C}\right)$.

\section{ALP}

Ten trials that included 335 patients reported data regarding this endpoint. Combination therapy with bezafibrate and UDCA was more effective than UDCA alone in reducing ALP level over a trial duration $>12$ months (SMD -2.33, $95 \% \mathrm{CI}-4.03$ to $-0.63 ; \mathrm{P}=0.007)$ and there was severe heterogeneity $\left(\mathrm{P}<0.001, I^{2}=94.7 \%\right)$. The results for a trial duration $<12$ months were similar (SMD -3.63 , 95\%CI -6.43 to $-0.84 ; \mathrm{P}=0.01)$, showing significant heterogeneity $(\mathrm{P}<0.001$, $\left.I^{2}=95.6 \%\right)($ Fig. $4 \mathrm{D} 1,4 \mathrm{D} 2)$.

\section{GGT}

This outcome was reported in 4 trials that included 146 patients. There was no significant difference between the monotherapy and combination therapy groups (SMD -1.29, $95 \% \mathrm{CI}-2.67$ to $0.08 ; \mathrm{P}=0.065)$. There was significant heterogeneity $\left(\mathrm{P}<0.001, I^{2}=90.7 \%\right)$ (Fig. $\left.4 \mathrm{E}\right)$.

\section{Albumin}

A total of 144 patients from 3 trials were analyzed for changes in albumin levels. There was no statistically significant difference between the 2 groups (SMD 0.35, 95\%CI -0.40 to $1.10 ; \mathrm{P}=0.358)$. There was severe heterogeneity $(\mathrm{P}=0.013$, $\left.I^{2}=76.9 \%\right)($ Fig. $4 \mathrm{~F})$.

Table 1 Baseline characteristics of the trials included in the meta-analysis

\begin{tabular}{|c|c|c|c|c|c|c|c|c|}
\hline Study & $\begin{array}{c}\text { Year of } \\
\text { publication }\end{array}$ & Type & $\begin{array}{l}\text { Treatment duration } \\
\text { (months) }\end{array}$ & $\begin{array}{l}\text { Bezafibrate } \\
\text { dose } \\
\text { (mg/day) }\end{array}$ & $\begin{array}{l}\text { UDCA dose } \\
\text { (mg/day) }\end{array}$ & $\begin{array}{l}\text { Combination } \\
\text { therapy (n) }\end{array}$ & $\begin{array}{c}\text { Monotherapy } \\
\text { (n) }\end{array}$ & $\begin{array}{l}\text { Mean age } \\
\text { (year) }\end{array}$ \\
\hline Corpechot et al [12] & 2018 & RCT & 24 & 400 & $900-1500$ & 50 & 50 & 53 \\
\hline Hosonuma et al [15] & 2015 & RCT & 96 & 400 & $600-900$ & 13 & 14 & 64 \\
\hline Lens et al [16] & 2014 & RCT & 3 & 400 & $900-1500$ & 28 & 28 & 53 \\
\hline Honda et al [11] & 2013 & RCT & 3 & 400 & 600 & 19 & 31 & 58 \\
\hline Takeuchi et al [20] & 2011 & RCT & 24 & 400 & 600 & 15 & 22 & 57 \\
\hline Hazzan et al [16] & 2010 & RCT & 24 & 400 & $900-1500$ & 8 & 8 & 64 \\
\hline Iwasaki et al [18] & 2008 & RCT & 12 & 400 & 600 & 12 & 10 & 54 \\
\hline Itakura et al [19] & 2004 & RCT & 6 & 400 & 600 & 9 & 7 & 57 \\
\hline Kanda et al [21] & 2003 & RCT & 6 & 400 & 600 & 11 & 11 & 56 \\
\hline Nakai et al [22] & 2000 & RCT & 12 & 400 & 600 & 10 & 13 & 58 \\
\hline
\end{tabular}

RCT, randomized controlled trial; UDCA, ursodeoxycholic acid 

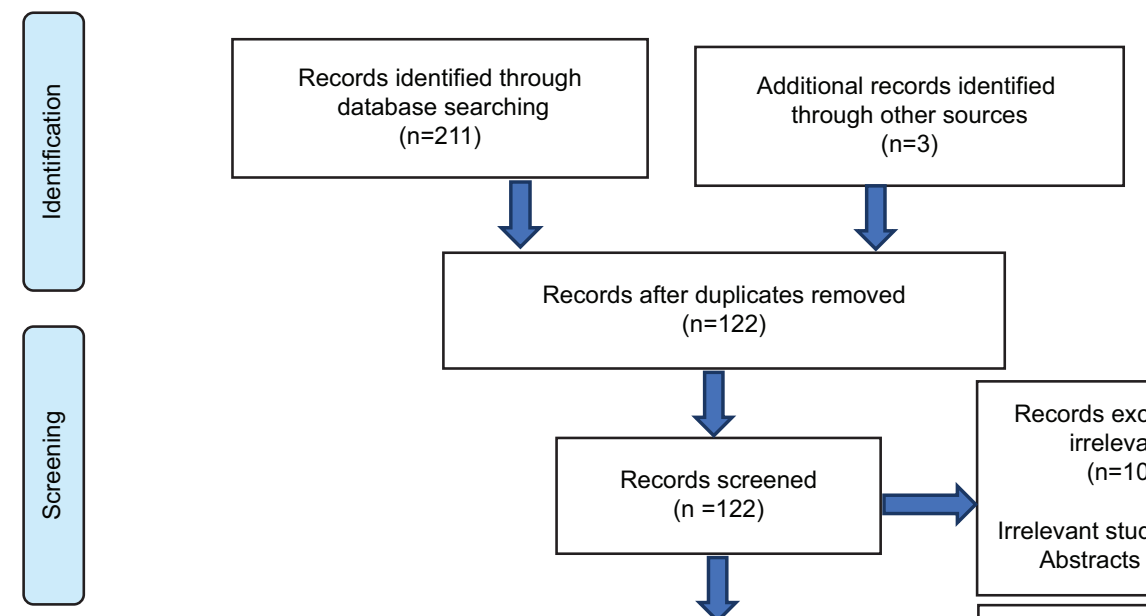

Records after duplicates removed $(n=122)$
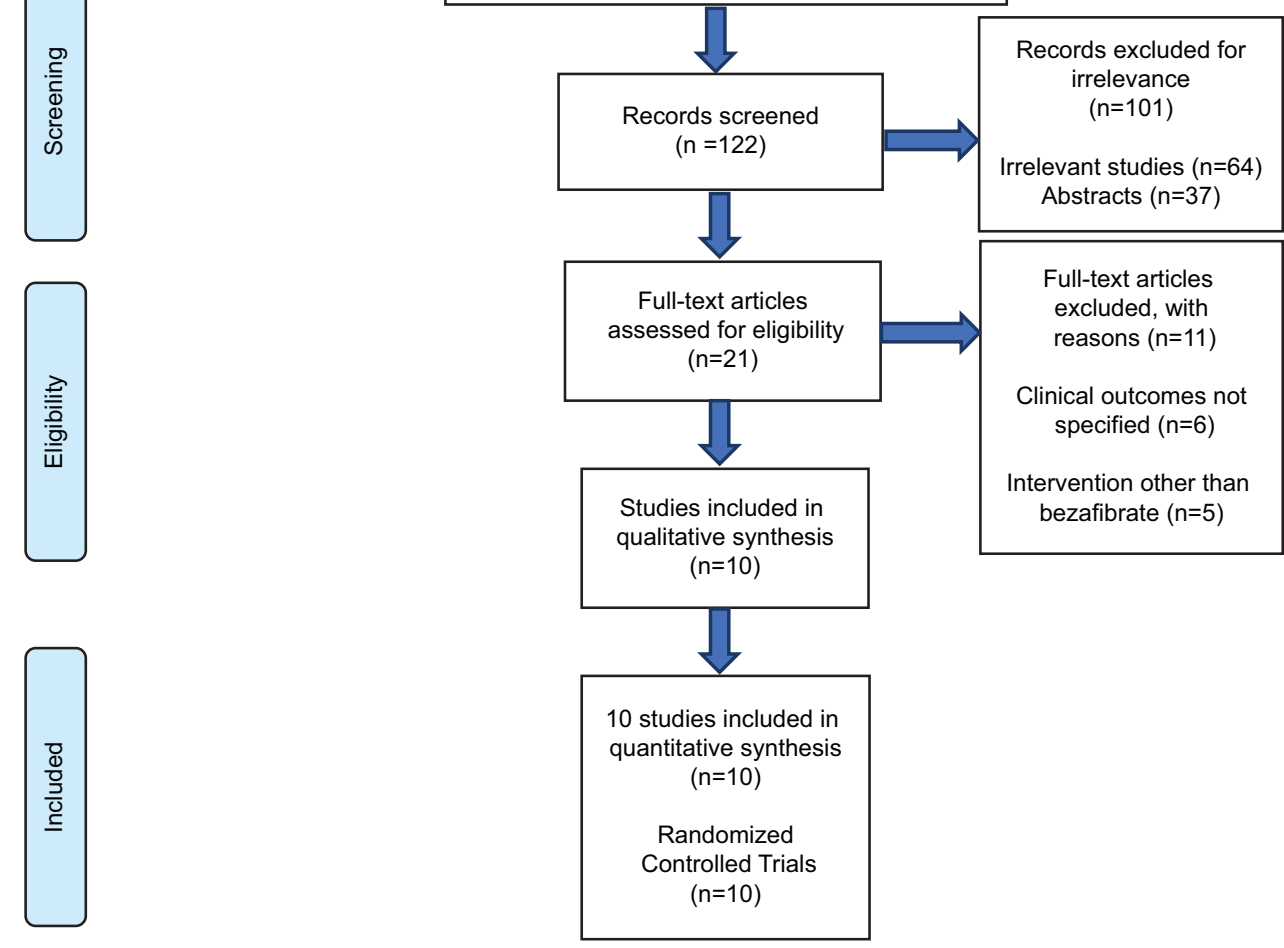

Figure 1 PRISMA diagram for selection of trials

\section{TG level}

Four trials that included 115 patients reported data regarding this endpoint. Combination therapy with bezafibrate and UDCA was more effective than monotherapy with UDCA in improving TG level (SMD $-0.80,95 \% \mathrm{CI}-1.41$ to $-0.19 ; \mathrm{P}=0.01)$. There was moderate heterogeneity ( $\left.\mathrm{P}=0.075, I^{2}=56.6 \%\right)$ (Fig. $\left.4 \mathrm{G}\right)$.

\section{IgM}

This endpoint was reported in 6 trials that included 199 patients. Combination therapy was more effective in lowering $\operatorname{IgM}$ as compared to monotherapy (SMD -1.48, $95 \% \mathrm{CI}-2.39$ to $-0.56 ; \mathrm{P}=0.002)$ and there was severe heterogeneity $\left(\mathrm{P}<0.001, I^{2}=82.5 \%\right)$ (Fig. $\left.4 \mathrm{H}\right)$.

\section{Cholesterol}

Five trials that included 196 patients reported a change in cholesterol levels. Combination therapy was more effective in lowering cholesterol compared to monotherapy (SMD -4.61,
95\%CI -7.34 to $-1.89 ; \mathrm{P}=0.001)$. There was severe heterogeneity $\left(\mathrm{P}<0.001, I^{2}=97.5 \%\right)$ (Fig. $\left.4 \mathrm{I}\right)$.

\section{Any adverse events}

Any adverse event was reported in 5 trials that included 160 patients. No statistically significant difference was found between the 2 groups (OR 2.05, 95\%CI 0.52-8.13; $\mathrm{P}=0.308$ ) and there was mild heterogeneity ( $\left.\mathrm{P}=0.196, I^{2}=33.8 \%\right)$ (Fig. $\left.5 \mathrm{~A}\right)$.

\section{CK levels}

Elevation of CK after initiation of therapy was analyzed in 3 trials that included 149 patients. In our meta-analysis no difference was found between UDCA monotherapy and combination therapy (SMD 4.44, 95\%CI 0.70-28.12; $\mathrm{P}=0.114$ ). There was no heterogeneity $\left(\mathrm{P}=0.906, I^{2}=0 \%\right)$ (Fig. $\left.5 \mathrm{~B}\right)$.

\section{Myalgias}

The incidence of myalgias after the initiation of therapy was reported in 3 trials that included 149 patients and there was 
no statistical difference between the 2 groups (OR 2.39, 95\%CI 0.85-6.74; $\mathrm{P}=0.098)$. There was no heterogeneity $(\mathrm{P}=0.967$, $I^{2}=0 \%$ ) (Fig. 5C).

\section{Pruritus}

Five trials that included 205 patients evaluated differences in pruritus incidence between monotherapy and combination therapy groups. There was no statistically significant difference between the two groups (OR 0.58, 95\%CI 0.16-2.08; $\mathrm{P}=0.406$ ). There was moderate heterogeneity $\left(\mathrm{P}=0.056, I^{2}=56.7 \%\right)$ (Fig. 5D).

\section{Fatigue}

No statistically significant difference was found between monotherapy and combination therapy groups in terms of fatigue, as reported in only one study that included 100 patients (OR 1.95, 95\%CI 0.76-5.01; $\mathrm{P}=0.164$ ). There was no heterogeneity $\left(\mathrm{P}=0.0, I^{2}=0 \%\right)$ (Fig. $\left.5 \mathrm{E}\right)$.

\section{Mortality}

Two trials that included 65 patients reported a change in mortality, but no statistical difference was found between the 2 groups ( $\mathrm{OR} 2.28,95 \% \mathrm{CI} 0.12-43.25 ; \mathrm{P}=0.582$ ). There was mild heterogeneity $\left(\mathrm{P}=0.189, I^{2}=42.1 \%\right.$ ) (Fig. $\left.5 \mathrm{~F}\right)$.

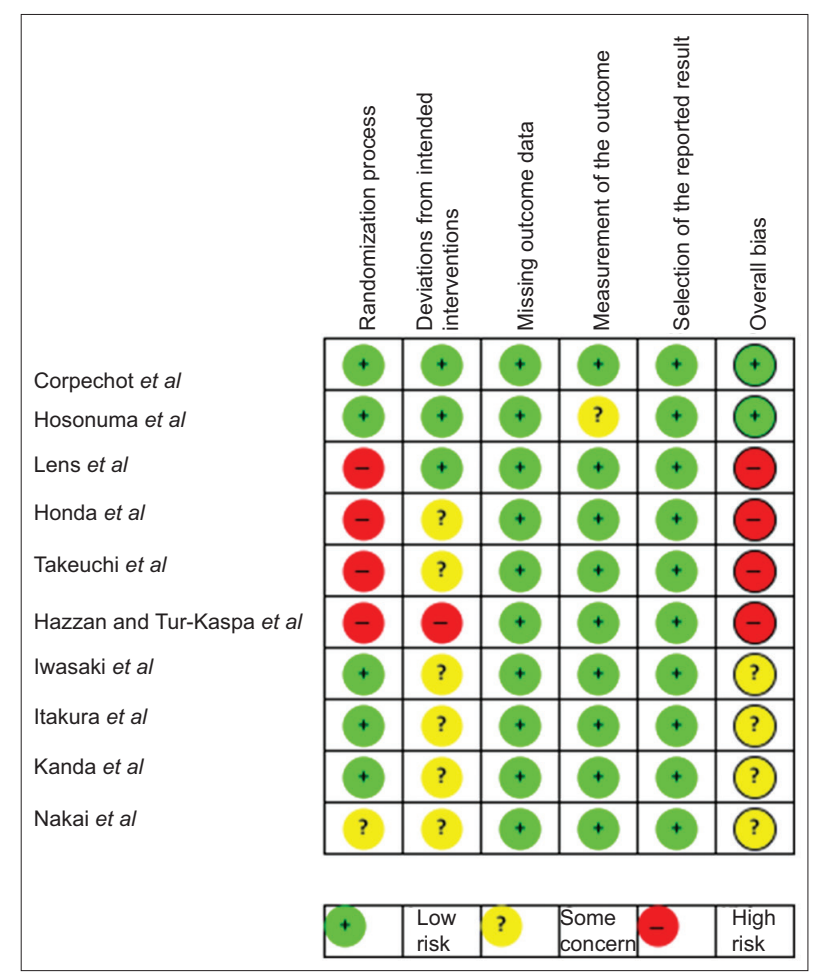

Figure 2 Risk of bias in included studies

+ indicates an increase, - indicates a decrease, and? indicates this is unclear

\section{Discussion}

Among the laboratory parameters studied, our analysis showed that there was a statistically significant reduction in ALT, ALP, TG, cholesterol, and IgM levels. The mechanisms via which bezafibrate influences these parameters are not completely understood. It has been suggested that the effects are driven by its action on PPAR- $\alpha, \beta / \delta$ and $\gamma$, with resulting upregulation of multidrug resistant gene 3 (MDR3). Decreased accumulation of cytotoxic bile acids within hepatocytes, via the downregulation of several proteins, including but not limited to cholesterol 7a-hydroxylase/sterol 27-hydroxylase, and its effects on the cytochrome P450 system might also contribute to its anti-cholestatic effects. The reductions in cholesterol and TG levels are probably caused by a combination of $\beta$-hydroxy $\beta$-methylglutaryl-CoA reductase inhibition and PPAR-induced cholesterol efflux from hepatocytes [11]. Through the same mechanism of action, we would have expected a decrease in bilirubin, AST, GGT, and albumin levels, but the changes were not a statistically significant in our analysis. These findings are similar to the analysis presented by Hosonuma et al, who failed to show a significant reduction in bilirubin, AST and albumin levels [15]. However, several studies have documented a statistically significant decrease in GTT levels [16-19]. It is documented that PPAR- $\alpha$ is present on T and B lymphocytes, which could explain the anti-inflammatory effects of bezafibrate, as suggested by the reduced levels of IgM [18].

There was no statistically significant association between bezafibrate use and an increase in side-effects such as pruritus, myalgia, fatigue or elevations of CK levels. In fact, there was a trend, although statistically not significant, towards an improvement in pruritus that has been reported individually in several trials. The mechanism for this relief is not entirely clear, but it could partly involve its anti-cholestatic effect. This is an important difference to note, as obeticholic acid, an alternative therapy to $\mathrm{PBC}$, is associated with significant pruritus [8]. The CK fluctuations are likely to be a consequence of bezafibrateinduced muscle injury. Corpechot et al reported elevated serum creatinine levels with bezafibrate use, which may be secondary to rhabdomyolysis causing elevation of both creatine kinase and myoglobin, inducing nephrotoxicity $[13,23]$. Other reported side effects are gastrointestinal discomfort (nausea, heartburn, diarrhea), arthralgia, myalgia, upper respiratory symptoms (nasopharyngitis, bronchitis), leg edema, polydipsia, and leg edema. These parameters were not reported in adequate numbers and therefore could not be analyzed.

Mortality was reported in only 2 trials and it was not a statistically significant outcome in our analysis. Our metaanalysis included trials with small sample sizes, only a few trials reported mortality, and moreover the trials we examined were constrained by short follow-up periods. The wide confidence intervals indicate the non-reliability of this finding; hence, larger trials will be needed to adequately assess the direct effect of bezafibrate on survival.

Variables of disease progression as assessed by histology, noninvasive measures of liver fibrosis (liver elastography) and prognostic scores were reported in only a few trials, limiting 


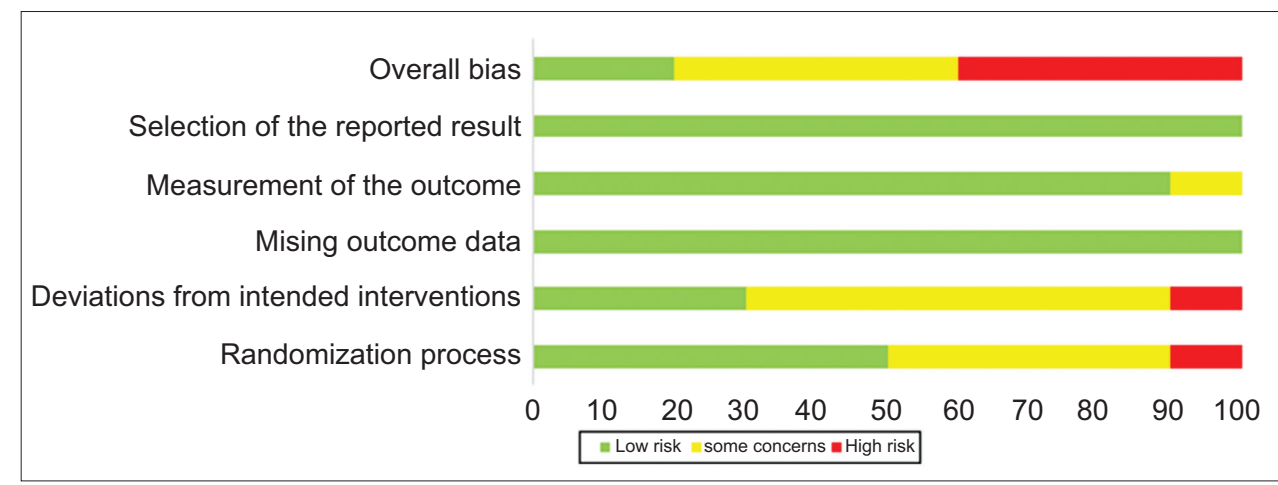

Figure 3 Risk of bias graph: review of authors' judgments regarding each risk of bias item presented as percentages across all included studies

Table 2 Meta-analysis of clinical events and changes in biochemical parameters in the included studies

\begin{tabular}{|c|c|c|c|c|c|}
\hline Outcome & $\begin{array}{l}\text { Number } \\
\text { of studies }\end{array}$ & $\begin{array}{l}\text { Number of } \\
\text { participants }\end{array}$ & Statistical method & Effect size & P-value \\
\hline Bilirubin & 5 & 164 & SMD (IV, random, 95\%CI) & $-0.77(-2.20$ to 0.65$)$ & 0.287 \\
\hline ALT & 3 & 142 & SMD (IV, random, 95\%CI) & $-2.04(-3.30$ to -0.79$)$ & 0.001 \\
\hline AST & 3 & 128 & SMD (IV, random, 95\%CI) & $-0.34(-1.76$ to 1.08$)$ & 0.639 \\
\hline ALP (duration $>12$ months) & 6 & 205 & SMD (IV, random, 95\%CI) & $-2.33(-4.03$ to -0.63$)$ & 0.007 \\
\hline ALP (duration $<12$ months) & 4 & 130 & SMD (IV, random, 95\%CI) & $-3.63(-6.43$ to -0.84$)$ & 0.01 \\
\hline GGT & 4 & 146 & SMD (IV, random, 95\%CI) & $-1.29(-2.67$ to 0.08$)$ & 0.065 \\
\hline Albumin & 3 & 144 & SMD (IV, random, 95\%CI) & $0.35(-0.40$ to 1.10$)$ & 0.358 \\
\hline TG & 4 & 115 & SMD (IV, random, 95\%CI) & $-0.80(-1.41$ to -0.19$)$ & 0.01 \\
\hline IgM & 6 & 199 & SMD (IV, random, 95\%CI) & $-1.48(-2.39$ to -0.56$)$ & 0.002 \\
\hline Cholesterol & 5 & 196 & SMD (IV, random, 95\%CI) & $-4.61(-7.34$ to -1.89$)$ & 0.001 \\
\hline Any adverse events & 5 & 160 & OR (M-H, random, 95\%CI) & 2.05 (0.52 to 8.13$)$ & 0.308 \\
\hline CK & 3 & 149 & SMD (IV, random, 95\%CI) & 4.44 (0.70 to 28.12$)$ & 0.114 \\
\hline Myalgia & 3 & 149 & OR (M-H, random, 95\%CI) & $2.39(0.85$ to 6.74$)$ & 0.098 \\
\hline Pruritus & 5 & 205 & OR (M-H, random, 95\%CI) & 0.58 (0.16 to 2.08$)$ & 0.406 \\
\hline Fatigue & 1 & 100 & OR (M-H, random, 95\%CI) & $1.95(0.76$ to 5.01$)$ & 0.164 \\
\hline Mortality & 2 & 65 & OR $(\mathrm{M}-\mathrm{H}$, random, $95 \% \mathrm{CI})$ & $2.28(0.12$ to 43.25$)$ & 0.582 \\
\hline
\end{tabular}

M-H, Mantel-Haenszel; CI, confidence interval; IV, inverse-variance; $M D$, mean difference; OR, odds ratio; ALT, alanine aminotransferase; AST, aspartate aminotransferase; $A L P$, alkaline phosphatase; GGT, gamma-glutamyltransferase; TG, triglyceride; CK, creatinine kinase

our analysis of the available data. An improvement in liver elastography in the patients receiving combination therapy was reported by 2 trials. Histological and fibrosis stage, along with activity grade on histology, did not vary significantly in a study by Corpechot et al, whereas significant differences were reported by Lens et al among patients receiving combination therapy $[12,16]$. Prognostic models, such as the Mayo risk score, have been used to estimate survival, but only 2 studies reported such findings. An improvement in the score was seen in both studies: the change was insignificant in a study by Takeuchi et al but significant according to Hosonuma et al $[20,15]$. The trials included in our analysis were completed prior to the validation of the GLOBE score, a prognostic model proposed by the Global PSC Study Group, which adequately predicts the transplant-free survival of patients being treated with UDCA [24].
Understanding the effect of bezafibrate on endpoints such as mortality, liver transplantation rates and histological improvements in liver biopsy are, of course, the most significant markers for this PBC therapy. However, the slow progression of the disease, the paucity of patients and the limited duration of studies make these hard endpoints difficult to obtain. We have evidence that the bilirubin and ALP reductions can predict outcomes such as liver transplantation and death $[25,26]$. Patients with ALP levels less than 2 times the upper limits of normal have an $84 \%$ survival rate at 10 years [25]. With these supporting studies, we can infer that the reductions in ALP achieved with combination therapy in patients who do not respond to UDCA alone can improve transplant-free survival and reduce death. 
A

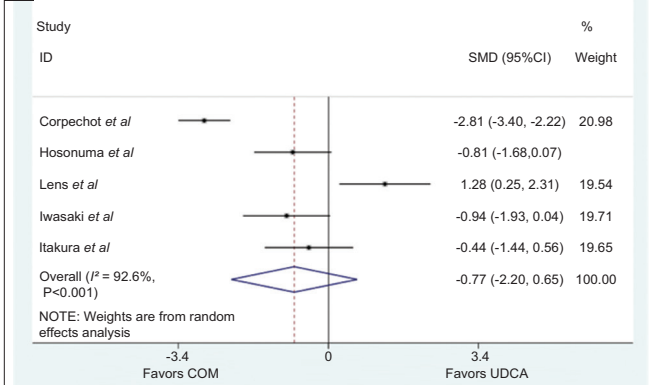

B

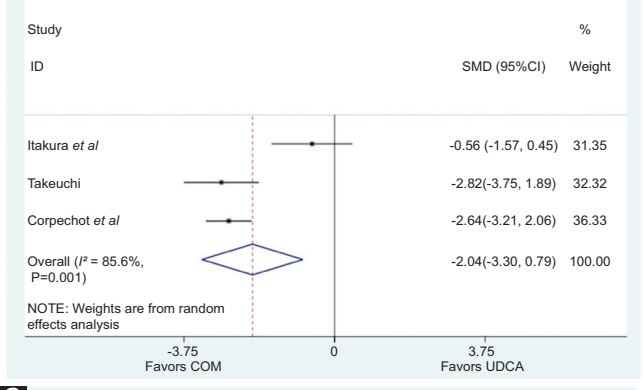

C

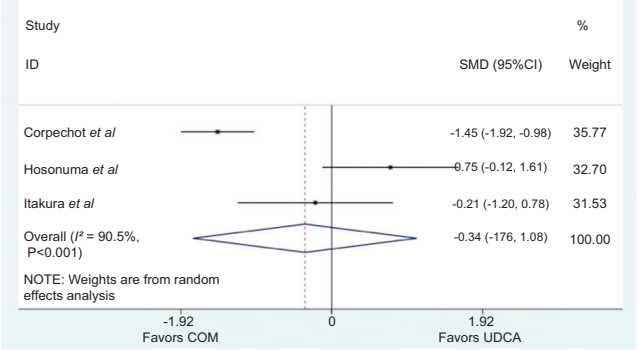

D1

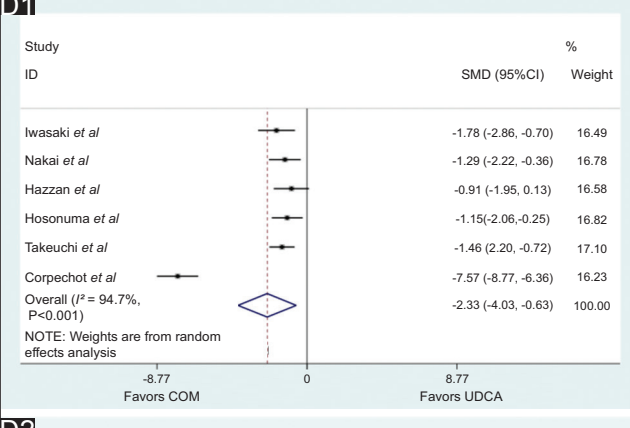

D2

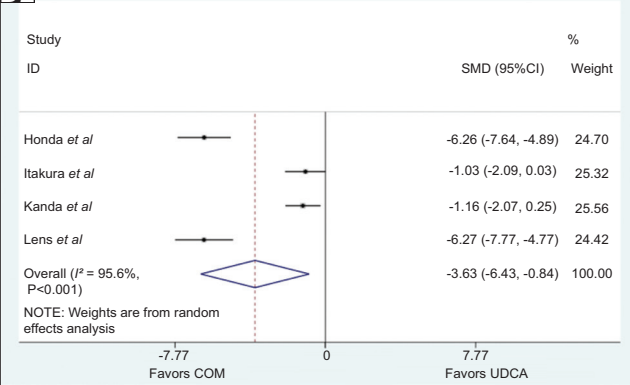

E

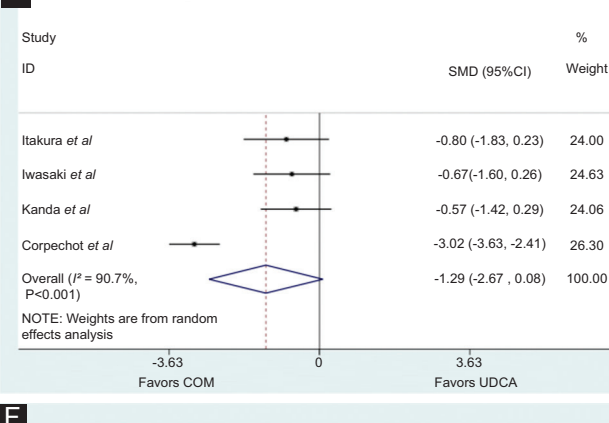

F

Study

ID

SMD (95\% CL) Weight

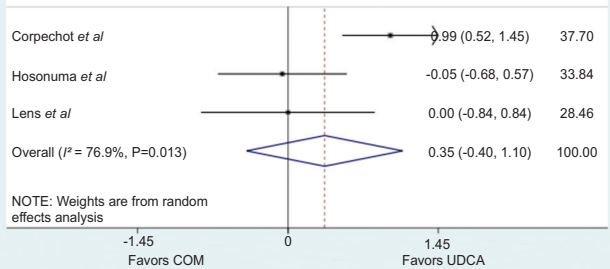

G

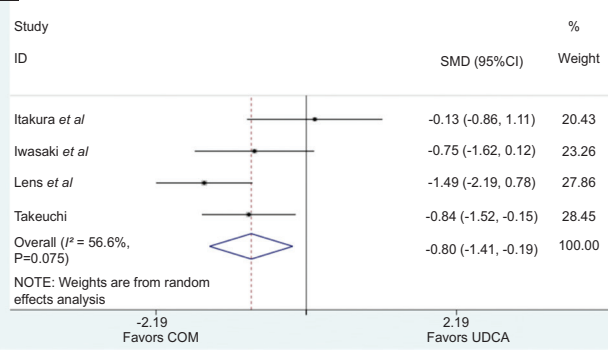

H

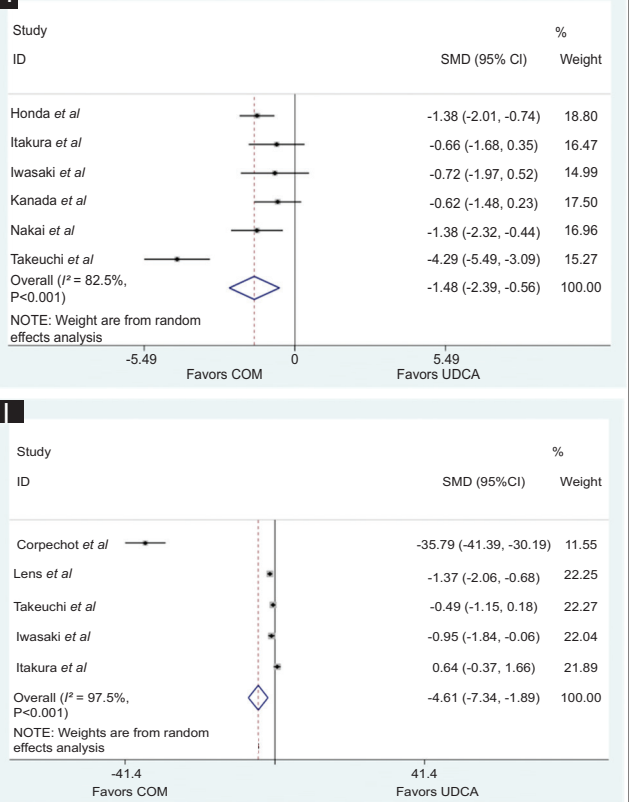

Figure 4 Biochemical parameters in PBC patients treated with UDCA versus combination therapy. (A) Bilirubin; (B) ALT; (C) AST; (D1): ALP (>12 months); (D2) (<12 months); (E) GGT; (F) Albumin; (G) TG; (H) IgM; (I) Cholesterol

$P B C$, primary biliary cholangitis; UDCA, ursodeoxycholic acid; ALT, alanine aminotransferase; AST, aspartate aminotransferase; ALP, alkaline phosphatase; GGT, gamma-glutamyltransferase; TG, triglyceride; COM, combination therapy; SMD, standard mean deviation; CI, confidence interval 


\section{A}

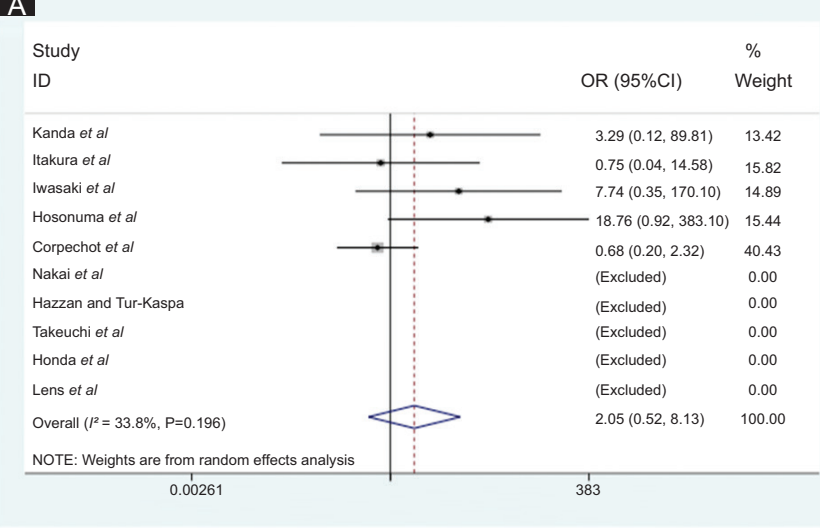

B
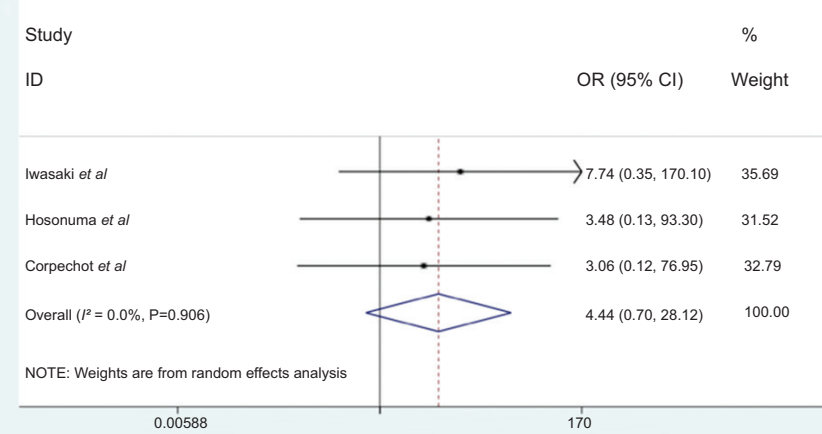

C
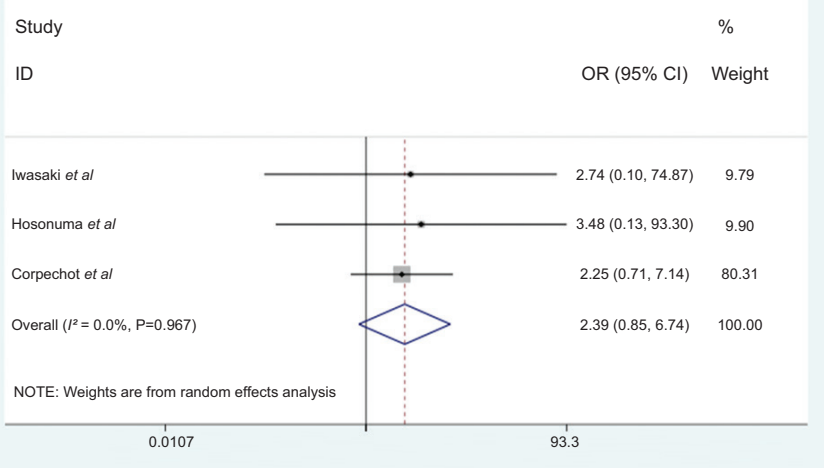

ID

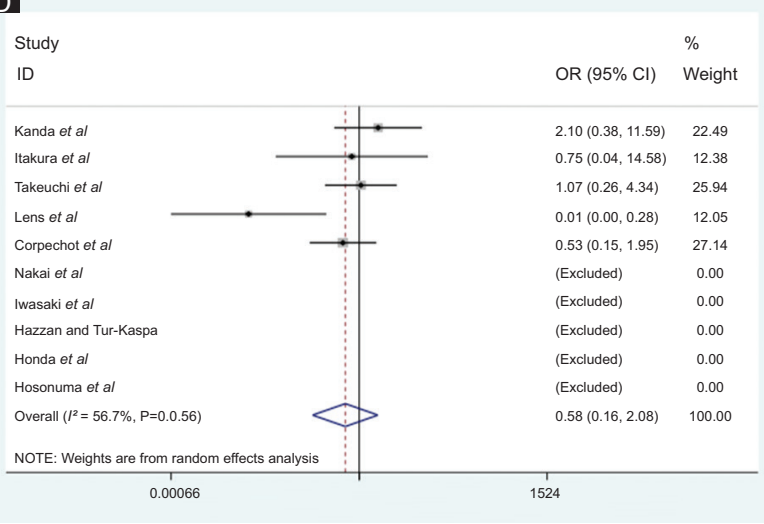

IE Study ID $\%$ OR $(95 \% \mathrm{Cl})$ Weight

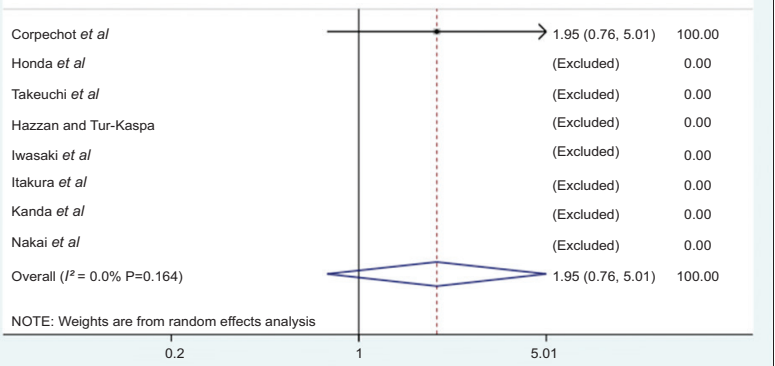

F Study ID OR $(95 \% \mathrm{Cl}) \quad$ Weight

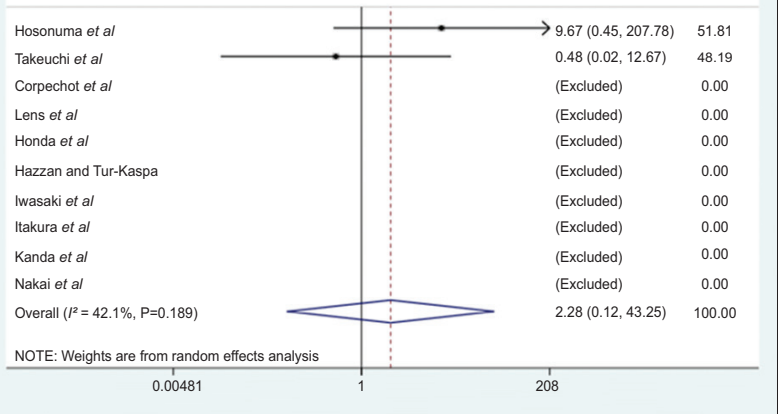

Figure 5 Adverse effects and mortality in PBC patients treated with UDCA versus combination therapy. (A) any adverse event; (B) CK; (C) myalgia; (D): pruritus; (E) fatigue; (F) mortality

PBC, primary biliary cholangitis; UDCA, ursodeoxycholic acid; COM, combination therapy; CK, creatinine kinase; OR, odds ratio; CI, confidence interval

This study had several limitations. First, we had a limited sample size of 369 patients, which reduced the power to detect a significant difference in some data points. We also did not have sufficient data to analyze important parameters such as histology, liver elastography, need for liver transplantation, mortality, quality of life and burden of side-effects, as most studies did not report this data. Lastly, these studies were also carried out over a short period of time; any long-term findings of bezafibrate would not be described in this metaanalysis.
In conclusion, a combination therapy of UDCA and bezafibrate is beneficial in improving biochemical parameters in patients with $\mathrm{PBC}$ who respond inadequately to UDCA alone. Using these biochemical parameters as a surrogate of survival, histological improvement and liver transplant-free periods, this meta-analysis suggests that bezafibrate may have a role in the management of such patients. However, larger trials with a more extensive follow up are needed to better characterize the long-term outcomes. 


\section{Summary Box}

\section{What is already known:}

- Ursodeoxycholic acid (UDCA) is the primary treatment for primary biliary cholangitis; however, $20 \%$ of patients respond inadequately

- Obeticholic acid has been approved as monotherapy in patients unable to tolerate UDCA because of side-effects; however, the significant nuisance associated with it is pruritus

- A combination therapy of bezafibrate and UDCA has been proposed for patients who respond inadequately to UDCA

\section{What the new findings are:}

- Combination therapy is associated with a statistically significant reduction in alanine aminotransferase, alkaline phosphatase, triglyceride, cholesterol, and IgM levels

- There was no statistically significant association between bezafibrate use and side-effects such as pruritus, myalgia, fatigue or elevation of creatinine kinase levels

- We propose that combination therapy with bezafibrate and UDCA may be a safe and effective therapy in patients who respond inadequately to UDCA alone

\section{References}

1. Poupon R. Primary biliary cirrhosis: a 2010 update. J Hepatol 2010;52:745-758.

2. Kaplan MM, Gershwin ME. Primary biliary cirrhosis. N Engl J Med 2005;353:1261-1273.

3. Parés A, Caballería L, Rodés J. Excellent long-term survival in patients with primary biliary cirrhosis and biochemical response to ursodeoxycholic Acid. Gastroenterology 2006;130:715-720.

4. Lindor KD, Therneau TM, Jorgensen RA, Malinchoc M, Dickson ER. Effects of ursodeoxycholic acid on survival in patients with primary biliary cirrhosis. Gastroenterology 1996;110:1515-1518.

5. Poupon RE, Balkau B, Eschwège E, Poupon R. A multicenter, controlled trial of ursodiol for the treatment of primary biliary cirrhosis. UDCA-PBC Study Group. N Engl J Med 1991;324:1548-1554.

6. Harms $\mathrm{MH}$, van B HR, Corpechot $\mathrm{C}$, et al. Ursodeoxycholic acid therapy and liver transplant-free survival in patients with primary biliary cholangitis. J Hepatol 2019 [Epub ahead of print]. doi: 10.1016/j.jhep.2019.04.001

7. Harms MH, Lammers WJ, Thorburn D, et al; Global PBC Study Group. Major hepatic complications in ursodeoxycholic acid-treated patients with primary biliary cholangitis: risk factors and time trends in incidence and outcome. Am J Gastroenterol 2018;113:254-264.

8. Kowdley KV, Luketic V, Chapman R, et al; Obeticholic Acid PBC Monotherapy Study Group. A randomized trial of obeticholic acid monotherapy in patients with primary biliary cholangitis. Hepatology 2018;67:1890-1902.
9. Nevens F, Andreone P, Mazzella G, et al; POISE Study Group. A placebo-controlled trial of obeticholic acid in primary biliary cholangitis. N Engl J Med 2016;375:631-643.

10. Corpechot C, Chazouilleres O, Rousseau A, et al. A 2-year multicenter, double-blind, randomized, placebo-controlled study on bezafibrate for the treatment of primary biliary cholangitis in patients with inadequate biochemical response to ursodeoxycholic acid therapy (Bezurso). J Hepatol 2017;66:LBO-101 (Abstract).

11. Honda A, Ikegami T, Nakamuta M, et al. Anticholestatic effects of bezafibrate in patients with primary biliary cirrhosis treated with ursodeoxycholic acid. Hepatology 2013;57:1931-1941.

12. Corpechot C, Chazouillères O, Rousseau A, et al. A placebocontrolled trial of bezafibrate in primary biliary cholangitis. N Engl J Med 2018;378:2171-2181.

13. Moher D, Liberati A, Tetzlaff J, Altman DG; PRISMA Group. Preferred reporting items for systematic reviews and metaanalyses: the PRISMA statement. PLoS Med 2009;6:e1000097.

14. Higgins JPT, Sterne JAC, Savović J, et al. A revised tool for assessing risk of bias in randomized trials In: Chandler J, McKenzie J, Boutron I, Welch V (editors). Cochrane Methods. Cochrane Database Syst Rev 2016:10(Suppl 1).

15. Hosonuma K, Sato K, Yamazaki Y, et al. A prospective randomized controlled study of long-term combination therapy using ursodeoxycholic acid and bezafibrate in patients with primary biliary cirrhosis and dyslipidemia. Am J Gastroenterol 2015;110:423-431.

16. Lens S, Leoz M, Nazal L, Bruguera M, Parés A. Bezafibrate normalizes alkaline phosphatase in primary biliary cirrhosis patients with incomplete response to ursodeoxycholic acid. Liver Int 2014;34:197-203.

17. Hazzan R, Tur-Kaspa R. Bezafibrate treatment of primary biliary cirrhosis following incomplete response to ursodeoxycholic acid. J Clin Gastroenterol 2010;44:371-373.

18. Iwasaki S, Ohira H, Nishiguchi S, et al; Study Group of Intractable Liver Diseases for Research on a Specific Disease, Health Science Research Grant, Ministry of Health, Labour and Welfare of Japan. The efficacy of ursodeoxycholic acid and bezafibrate combination therapy for primary biliary cirrhosis: A prospective, multicenter study. Hepatol Res 2008;38:557-564.

19. Itakura J, Izumi N, Nishimura Y, et al. Prospective randomized crossover trial of combination therapy with bezafibrate and UDCA for primary biliary cirrhosis. Hepatol Res 2004;29:216-222.

20. Takeuchi Y, Ikeda F, Fujioka S, et al. Additive improvement induced by bezafibrate in patients with primary biliary cirrhosis showing refractory response to ursodeoxycholic acid. J Gastroenterol Hepatol 2011;26:1395-1401.

21. Kanda T, Yokosuka O, Imazeki F, Saisho H. Bezafibrate treatment: a new medical approach for PBC patients? J Gastroenterol 2003;38:573-578.

22. Nakai S, Masaki T, Kurokohchi K, Deguchi A, Nishioka M. Combination therapy of bezafibrate and ursodeoxycholic acid in primary biliary cirrhosis: a preliminary study. Am J Gastroenterol 2000;95:326-327.

23. Charach G, Grosskopf I, Rotmensch HH, Kitzis I, Weintraub M. Bezafibrates cause moderate, reversible impairment in renal function in patients without prior renal disease. Nephron Clin Pract 2005;100:c120-c125.

24. Lammers WJ, Hirschfield GM, Corpechot C, et al; Global PBC Study Group. Development and validation of a scoring system to predict outcomes of patients with primary biliary cirrhosis receiving ursodeoxycholic acid therapy. Gastroenterology 2015;149:1804-1812.

25. Lammers WJ, van Buuren HR, Hirschfield GM, et al; Global PBC Study Group. Levels of alkaline phosphatase and bilirubin are surrogate end points of outcomes of patients with primary biliary cirrhosis: an international follow-up study. Gastroenterology 2014;147:1338-1349.e5.

26. Silveira MG, Brunt EM, Heathcote J, Gores GJ, Lindor KD, Mayo MJ. American Association for the Study of Liver Diseases endpoints conference: design and endpoints for clinical trials in primary biliary cirrhosis. Hepatology 2010;52:349-359. 